\title{
Miranda
}

Revue pluridisciplinaire du monde anglophone /

Multidisciplinary peer-reviewed journal on the English-

speaking world

$10 \mid 2014$

Images on the Move: Circulations and Transfers in film

\section{Circulation dans Cars 2 (John Lasseter - Brad Lewis, 2011) et Drive (Nicolas Winding Refn, 2011)}

\section{Yann Roblou}

URL : http://journals.openedition.org/miranda/6270

DOI : $10.4000 /$ miranda.6270

ISSN : 2108-6559

Éditeur

Université Toulouse - Jean Jaurès

\section{Référence électronique}

Yann Roblou, "Circulation dans Cars 2 (John Lasseter - Brad Lewis, 2011) et Drive (Nicolas Winding Refn, 2011) », Miranda [En ligne], 10 | 2014, mis en ligne le 23 février 2015, consulté le 16 février 2021 URL : http://journals.openedition.org/miranda/6270 ; DOI : https://doi.org/10.4000/miranda.6270

Ce document a été généré automatiquement le 16 février 2021.

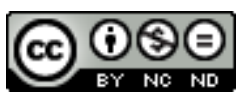

Miranda is licensed under a Creative Commons Attribution-NonCommercial-NoDerivatives 4.0 International License. 


\title{
Circulation dans Cars 2 (John Lasseter - Brad Lewis, 2011) et Drive (Nicolas Winding Refn, 2011)
}

\author{
Yann Roblou
}

1 Phénomène industriel puis, de manière quasi-concomitante, phénomène culturel, la voiture tient une place fondamentale dans l'univers matériel et symbolique de l'Amérique. Depuis sa première apparition dans les rues des villes nord-américaines au début du siècle dernier puis la mise à portée de tous les citoyens par Henry Ford de sa célèbre Model $\mathrm{T}$, l'automobile a rapidement dépassé sa fonction de simple moyen de transport pour acquérir celle de signe extérieur de statut social pour son propriétaire.

$\mathrm{Au}$ lendemain de la seconde Guerre Mondiale, et particulièrement dans les années cinquante, les adolescents peuvent ainsi devenir acquéreurs de leur propre voiture, cette dernière s'installant dès lors de manière pérenne dans ce qu'il convient d'appeler la culture américaine. Et pas simplement dans la rue, comme en témoignent ces quelques exemples venus du monde de la musique : de l'emblématique Car Song (1947) de Woodie Guthrie au Little Deuce Coupe (1963) des Beach Boys, du tube des bien nommés Cars Drive (1984) jusqu'au Let Me Ride (1993) de Dr Dre, en passant par les célèbres Mustang Sally (1966) de Wilson Pickett, Mercedes Benz (1970) de Janis Joplin, One Piece at a Time (1976) de Johnny Cash ou Car Wash (1976) de Rose Royce, ce sont autant d'hymnes à la gloire de ce mythe moderne qu'est l'automobile.

3 Le cinéma n'est évidemment pas en reste avec, dès le début du vingtième siècle, ses stars qui se font volontiers photographier en compagnie de leur(s) véhicule(s) ${ }^{1}$. Mais on songe bien sûr également aux acteurs (James Dean ou Steve McQueen, pour ne citer qu'eux) dont le sort est intimement lié, à la ville ou à l'écran, au monde de l'automobile. Et il est même tout à fait significatif que, parfois, la voiture prenne plus de place que certains comédiens qui ne servent alors que de faire-valoir aux engins. Citons en exemple Judy Carlson, Lisa Herman et Suzanne Somers qui apparaissent toutes trois au générique d'American Graffiti (George Lucas, 1973) mais sont désignées, respectivement, comme étant "Girl in Studebaker », "Girl in Dodge » et "Blonde in T-Bird». On 
n'oubliera pas que la voiture peut, à l'occasion, devenir la star du film, dans des genres aussi divers que la comédie familiale estampillée Walt Disney (la série des Herbie/ Coccinelle $^{2}$ ) ou le film d'horreur (le Christine de John Carpenter, 1983).

4 Les deux films qui vont être évoqués s'inscrivent dans cette phénoménologie culturelle de la voiture tout en offrant des singularités que la notion de circulation met adéquatement en lumière. En effet, et bien qu'ils soient très différents, Cars 2 et Drive illustrent un nombre important de types de circulations, que ce soit au sens le plus littéral comme de façon plus figurée. C'est donc autour de cette notion que je me propose d'aborder les deux films en opérant des allers-retours qui pourront permettre des rapprochements (comparatifs et distinctifs) entre les deux œuvres. De manière peut-être moins anecdotique, les deux longs-métrages offrent également des exemples de circulation du sens qui méritent que l'on s'y attarde. Enfin, les éléments qui composent Cars 2 et Drive - notamment l'intrigue et les personnages qui y sont développés - permettent de mettre en évidence certaines structures plus profondes et des pistes de lecture de l'image qui est donnée de l'Amérique du début du vingt-etunième siècle.

5 Afin de faciliter la tâche au lecteur, un rapide synopsis des deux films peut s'avérer utile.

6 Cars 2: À peine revenu à Radiator Springs pour un moment de détente après sa quatrième victoire dans la Doc Hudson Cup, Lightning (Flash en v.f.) McQueen doit reprendre le chemin des circuits pour participer à une série d'épreuves internationales opposant les meilleurs véhicules des principales compétitions automobiles. À l'origine de l'événement, Sir Mile Axelrod, un milliardaire qui s'est récemment converti à la production de carburant "vert", l'Allinol, qu'il entend promouvoir lors des trois courses en le fournissant aux participants. Tow Mater (Martin de ce côté-ci de l'Atlantique), la dépanneuse amie de McQueen, fait partie du voyage mais doit bientôt, à son corps défendant, aider un agent des services secrets britanniques à déjouer une machination internationale visant à nuire à Axelrod. En utilisant une fausse caméra de télévision, les lemons (les voitures aux défauts cachés), menées par le Professeur Zündapp et un mystérieux commanditaire, font exploser l'un après l'autre les moteurs des bolides qui se servent de l'Allinol. Une fois le complot déjoué et les malfrats appréhendés, tous les concurrents se retrouvent à Radiator Springs pour une ultime course « pour le plaisir».

7 Drive : Los Angeles. Mécanicien et cascadeur le jour, Driver (Ryan Gosling) travaille comme convoyeur pour la pègre la nuit. Lorsqu'il rencontre Irene (Carey Mulligan), sa voisine de palier dans l'immeuble où il vient d'emménager, sa vie bascule. Pour venir en aide au mari de celle-ci tout juste libéré de prison, il lui sert de chauffeur pour un casse qui tourne mal. Il échappe à un guet-apens qui devait permettre à Nino (Ron Perlman), l'un des patrons de la mafia locale, de mettre la main sur un million de dollars appartenant à la mafia de la côte est. Après avoir informé Bernie (Albert Brooks), son supérieur, de la situation, Nino et lui décident d'éliminer tous ceux qui sont impliqués dans l'affaire. C'est sans compter sur Driver qui, pour protéger Irene, les tue l'un après l'autre avant de disparaître, grièvement blessé par Bernie.

8 En surface, Cars 2 et Drive fourmillent d'illustrations de ce que l'on entend généralement par circulation et qui est bien évidemment conditionné par la présence centrale de la voiture. Ne serait-ce que parce que cette dernière sert de référent explicite au film de 
Lasseter et Lewis, et que le terme qui donne son titre à celui de Refn est intimement (mais pas exclusivement) lié à la conduite.

Quant aux personnages des deux fictions, ils s'imposent comme des vecteurs de circulation par excès pourrait-on dire. Ceux qui ont vu l'un ou l'autre des films d'animation produits par le studio Pixar savent que l'univers des protagonistes de Cars est singulier et caractéristique : toute figure humaine en est absente. Le spectateur est plongé dans une recréation du monde défini par le "véhiculo-centrisme " - si l'on autorise ce néologisme. Certes les voitures sont dotées de qualités humaines (des yeux, une bouche, des sentiments) propres à l'anthropomorphisme hérité des films d'animation produits par Disney, mais il est intéressant d'observer le systématisme avec lequel tout est transformé pour être à l'image de la voiture. Ainsi les décors montagneux de Radiator Springs sont modelés sur les lignes des carrosseries des Chevrolet, Cadillac et autres Pontiac. Les noms propres sont eux aussi adaptés (l'un des revendeurs d'accessoires de la bourgade où résident les protagonistes s'appelle "Casa Della Tires"), voire modifiés (l'emblématique Big Ben anglais est travesti en Big Bentley). L'univers même des films Pixar devient source d'auto-référence puisqu'au programme du drive-in s'affiche "The Incredimobiles", clin d'œil à une autre production maison : The Incredibles/Les Indestructibles (Brad Bird, 2004). De fait, tout en restant familier dans ses références pour le spectateur, le monde de Cars 2 acquiert une véritable autonomie, et repérer les distorsions subies par cette étonnante représentation du monde peut devenir source de plaisir métafictionnel. ${ }^{3}$

De son côté, Drive nous invite à suivre la trajectoire d'un personnage dont la désignation et la fonction semblent interchangeables. Il est intrigant de noter, toutefois, que son nom, Driver, n'est jamais prononcé tout au long du film mais apparait, associé au nom de l'acteur principal, simplement dans la distribution des rôles. C'est donc uniquement par sa présence derrière un volant qu'il acquiert une identité, voire une existence. Et il n'est pas anodin que la démultiplication de ses activités ${ }^{4}$ établisse un rapport à la voiture pour le moins extraordinaire.

11 Par ailleurs, la structure des deux films met en jeu la circulation (au sens de mouvement, de déplacement physique), mais sur des modes fondamentalement opposés. En contraste avec la construction volontairement fermée de Cars 2 (quand bien même les personnages se déplacent autour du globe, on part de Radiator Springs pour mieux y revenir), celle de Drive propose une dynamique plus complexe, dont l'ouverture est fondée sur l'indétermination (on ne sait d'où vient Driver, ni précisément depuis combien de temps il hante les rues de Los Angeles). De plus, une fois arrivée à son terme, cette dynamique narrative demeure incertaine : le spectateur est laissé dans l'expectative concernant l'avenir du protagoniste. Est-il blessé mortellement? Va-t-il redémarrer une nouvelle vie ? Le dernier plan du film n'apporte aucune réponse. A l'instar de ce qu'il met en place dans la conclusion de ses précédents films, Refn laisse le spectateur imaginer ce que le protagoniste peut/va devenir. Cette incertitude crée une tension que le cinéaste espère durable dans l'expérience que l'on peut faire de son cinéma ${ }^{5}$. A l'inverse, le public principal auquel s'adressent les productions Pixar s'attend à un retour à la stabilité du cosmos après le chaos (contrôlé) de ce qui a été montré. Et l'on serait bien en peine d'imaginer une filiale de Disney n'offrant pas une vision rassurante du monde à la fin d'un de ses films.

12 Complétant, tout en se démarquant de leur construction globale, les deux films mettent en œuvre les modes de circulation automobiles dans des décors qui induisent des 
lectures et des perceptions de l'espace très différentes : on est en vase clos dans le longmétrage de Refn, comme en témoigne le premier plan du film, qui photographie en gros plan une carte du centre ville de Los Angeles. Par ailleurs, tout ce qui se déroule dans la diégèse est confiné à cet espace centripète : les seuls plans de très grand ensemble sont ceux du business district, pris dans l'extrême plongée d'une vue aérienne. Et les deux scènes qui ne se déroulent pas dans le centre ville ont comme décor, d'une part, les collines et, de l'autre, l'océan - éléments géographiques et topographiques qui s'imposent comme des frontières infranchissables et repoussent toujours le protagoniste vers le cœur de ténèbres du centre, le downtown au nom évocateur, dans la langue anglaise, d'une progressive descente aux enfers. À l'inverse, le film de Lasseter et Lewis démultiplie les espaces puisque le spectateur est amené à suivre les compétitions opposant des voitures de course de plusieurs nationalités sur les circuits du Japon et de l'Italie, de l'Angleterre et des Etats-Unis. De plus, les tribulations aventureuses de Mater nous permettent tantôt d'accompagner, tantôt de précéder les diverses courses automobiles en utilisant des moyens de transports qui accentuent encore l'impression d'ouverture et de délocalisation. Ainsi, voyager en jet privé ou en train à grande vitesse donnent à la dépanneuse devenue espion amateur l'occasion de se rendre, outre les pays déjà cités, en France et dans une ville imaginaire, Porta Corsa, qui est un composite de Nice, Monaco et Portofino.

13 Enfin, si l'on considère leur scansion respective, il faut souligner que la circulation prend une dimension singulièrement commune dans les deux films. S'intégrant dans un schéma dramatique parfaitement classique, ils sont en effet articulés autour de trois moments forts. Dans un cas (Cars 2), trois courses doivent désigner le meilleur véhicule du monde. Vient s'y ajouter une quatrième, qui prend fonction de coda : dans la rivalité qui oppose McQueen à Francesco Bernoulli (une F1 italienne vantarde et fanfaronne), chacun des deux concurrents remporte une victoire et la dernière course officielle est annulée. Tout se joue donc sur un ultime défi non homologué, sans journaliste ni public - si ce n'est la population de Radiator Springs, lieu qui s'impose décidément comme le centre du monde de l'automobile de course...

Chez Refn, la progression dramatique s'appuie sur trois courses-poursuites aux enjeux thématiques et esthétiques très différents : la première met en place les exceptionnels talents de conducteur de Driver. Sans s'appuyer sur un montage haletant ni sur des effets pyrotechniques spectaculaires, la séquence est essentiellement filmée de l'intérieur du véhicule, ce qui établit et conditionne notre rapport avec le protagoniste. La seconde montre ce dernier en difficulté après le braquage raté. Il est pris en chasse par des inconnus dans une puissante voiture et ne doit son salut qu'à son sang froid et son expérience. Cette séquence fait alterner plans intérieurs et extérieurs. En n'insérant que quelques plans plus larges dans un ensemble défini par la prépondérance des plans plus rapprochés dans lesquels la bande-son laisse une place presqu'exclusive aux bruits des moteurs, le cinéaste laisse ainsi une part essentielle à la tension dramatique. La dernière course-poursuite est plus brève que les précédentes. Elle met en scène l'exécution presque clinique de Nino par Driver. Les plans sont plus amples dans leur durée et dans le champ visuel qu'ils couvrent, mais, de manière paradoxale, c'est la réaction des poursuivis qui prévaut dans cette séquence nocturne à l'atmosphère glaciale.

15 On peut lire dans la progression ternaire de Cars 2 la volonté de ne pas favoriser l'un ou l'autre des deux rivaux (qui sont de toute façon de force et d'orgueil équivalents), voire 
d'insister indirectement sur un dépassement de la compétition. D'une part, en une sorte de pied de nez signifiant clairement que l'intérêt du film est ailleurs que dans la concurrence, nous n'assistons pas à la dernière course. D'autre part, les premiers tours de roues laissent à penser que ni McQueen ni Bernoulli ne prendront la première place puisque c'est le seul personnage qui ne conçoit la vitesse que comme divertissement, Mater, qui est en tête au moment où le générique de fin intervient, grâce aux réacteurs offerts par les espions anglais. Chez Refn, le rapport entre les trois moments est tout autre: la maestria initiale est remplacée par l'instinct de survie puis par le côté le plus mortifère de Driver. L'impact de cette dernière facette modifie la perception du spectateur : c'est une confirmation de la part sombre du personnage qui est exposée.

Comme on peut le constater, la circulation des protagonistes n'est pas simplement une affaire de péripéties. Elle conditionne des points de vue qui établissent autant de variations esthétiques et qui interrogent dans le même temps la notion clé de l'identité des protagonistes. Lorsque s'ouvre Cars 2, les auteurs du film nous plongent dans un univers qui n'a pas de rapport avec celui du premier volet. Il y a de nouveaux personnages et une intrigue d'espionnage est immédiatement mise en place: une voiture répondant au nom de Flinn McMissile surprend un complot concernant l'exploitation d'un gisement de pétrole en plein océan. Sur le point d'être fait prisonnier, McMissile feint de disparaître. En réponse à la question rhétorique de l'un des méchants (le Professor Zündapp) qui conclut cette séquence d'introduction : «With McMissile gone, who's going to stop us now ?» (« Avec la disparition de McMissile, qui va pouvoir se mettre en travers de notre route ?»), un cut de contraste abrupt nous propulse sur une route près de Radiator Springs. Et l'on découvre Mater la dépanneuse qui semble directement lui répondre en clamant haut et fort : «Mater. Tow Mater. Is here to help you!» («Martin. Martin la dépanneuse. À votre service !») ${ }^{7}$ Ce que cette association née du montage signale, comme par anticipation, c'est que Mater a changé de statut: de faire-valoir de Lightning McQueen dans le film précédent, il semble s'imposer d'emblée comme le personnage principal du présent opus.

On peut sans doute justifier contextuellement les choix dramatiques et narratifs opérés par la production : les deux décadrages ont permis de renouveler la franchise (introduction de nouveaux personnages, intrigue radicalement différente, internationalisation des décors...) tout en préservant ce qui faisait en partie le sel du premier épisode (aspect plus conventionnel, thématiquement et esthétiquement, du film de course automobile).

18 Confirmant la prééminence du personnage, le lien entre ces deux pôles n'est autre que Mater. À l'origine de la participation de McQueen dans la course au titre de champion du monde automobile toute catégorie, il est aussi invité à faire partie de l'écurie qui suit le bolide américain autour du monde. Et c'est de manière fortuite qu'il prend part au démantèlement du vaste complot international.

Ce que révèle le déroulement $\mathrm{du}$ film, c'est que la dépanneuse se caractérise à la fois par son en-deçà (son apparence déglinguée, son accent et son phrasé du terroir, son évidente ignorance des conventions culturelles d'autres pays, ses maladresses diverses - bref, tout ce par quoi il contraste avec les autres personnages) et son au-delà (Mater est véritablement le héros du film: c'est lui qui dénoue l'intrigue grâce à ses connaissances en matière de mécanique automobile, faisant dans le même temps preuve de ressources insoupçonnées - quand bien même il doit faire face à de redoutables adversaires ou à l'incompréhension de ses amis... - le tout sans se départir 
de son incurable candeur). Ainsi, le travail d'écriture des scénaristes (Lasseter et Lewis, mais surtout Dan Fogelman et Ben Queen) a remarquablement fait évoluer le personnage pour en faire un avatar réussi du « héros malgré lui », qui, pris dans une histoire aux enjeux qui le dépassent, synthétise les qualités de l'individu américain ordinaire, naïf et plein de ressorts.

$C^{\prime}$ 'est bien la tension entre ces deux extrêmes qui rend possible la circulation de son identité, à la fois diégétiquement et extradiégétiquement : on le croit stupide, c'est un expert infaillible dans son domaine ; on le considère comme un espion alors qu'il ne cesse de revendiquer son statut de dépanneuse. Pour le spectateur, qui n'est pas abusé par les problèmes de perception qui sont l'apanage des autres personnages de la fiction, les qualités (humaines est-on tenté de dire) de cœur de Mater sont évidemment ce qui fonde sa permanence.

Dans le cas de Drive, ainsi que nous l'avons évoqué, tout tourne autour des identités multiples liées aux diverses fonctions du protagoniste. Ainsi, le plan inaugural du film lui confère une présence désincarnée, comme le signifie le changement de statut de sa voix qui, d'abord en off, devient over. L'ambiguïté pour le spectateur tient dans son ignorance du statut du personnage : sa voix dit-elle son omniscience (et ce qui va suivre n'est que la narration actualisée de ce qui lui est arrivé à un moment, dans son passé) ou une interaction intradiégétique? Le flottement est entretenu par les premiers mots prononcés qui déroutent par leur aspect péremptoirement factuel ${ }^{8}$, effet amplifié par l'image qui s'ouvre progressivement sur une carte routière du centre ville, sur laquelle on voit des notes, flèches et indications marquées au crayon. La caméra poursuit son trajet panoramique latéral et nous permet de découvrir une figure humaine de dos, qui s'adresse à un interlocuteur au téléphone. Il nous faut attendre la séquence suivante pour que ce corps acquière un visage. Cette présentation dilatoire fait de Driver une sorte de maitre de jeu (les instructions qu'il donne ici pour la première fois - et qui seront reprises par la suite - étayent cette hypothèse) aux allures fantomatiques (la mobilité de la caméra couplée à la fluidité du montage ne sont pas étrangères à cet effet) pour qui les règles de fonctionnement de la circulation routière (tant sur le papier - la carte - que sur le terrain) n'ont aucun secret. Ce qu'il démontre dans la séquence suivante en organisant avec succès la fuite de deux cambrioleurs en se livrant à une sorte de jeu du chat et de la souris avec les forces de l'ordre. Sa maitrise du terrain se combinant à une connaissance intime de l'ombre et des recoins de la cité donne l'avantage à Driver, pourtant désavantagé face à la démultiplication des ressources (voitures, hélicoptère) de la police.

Néanmoins, il est une chose qu'il ne peut maîtriser : l'impact de sa rencontre avec Irene (Carey Mulligan). On a pu écrire, à l'occasion de la sortie du film, que Nicolas Winding Refn en avait fini avec les racines kubrickiennes de son inspiration. Il n'en demeure pas moins que, comme chez son modèle, c'est le hasard (le sort, le destin, la fatalité...) d'une rencontre qui bouleverse la mécanique parfaitement rodée de l'existence de Driver. En voulant aider le mari de sa voisine de palier à s'acquitter d'une dette contractée en prison, le jeune homme solitaire (il n'est pas impossible de lire à travers lui un constat sociétal sur l'extrême solitude des individus dans une grande métropole comme Los Angeles) et discret sort de sa réserve et, par là même, s'attire les foudres d'un parrain de la Mafia locale. Cette rupture de la discipline qu'il s'est jusqu'alors imposée entraîne un changement de statut. De loner (par choix peut-être tout autant que par nécessité : on ne sait rien de son passé), il devient littéralement protecteur de la veuve et de 
l'orphelin. Et il n'est pas inintéressant de noter que, d'une part, le hasard d'une rencontre fait de Driver une autre figure du «héros malgré lui». D'autre part, toutefois, ce bouleversement s'avère être moins positif que pour Mater puisqu'il est associé, à la manière de ce qu'on trouve dans les fictions mises en scène par Stanley Kubrick, à une dimension plus sombre et plus violente du protagoniste. La violence qui fait partie de l'univers de Driver semble tout d'abord lui être extérieure: homme de l'ombre et du retrait, il est toujours caché derrière quelque chose (un masque lorsqu'il est cascadeur) ou quelqu'un (Shannon, le patron du garage, interprété par Bryan Cranston) et la charge de violence est déplacée sur les personnages qui l'entourent (les cambrioleurs auxquels il loue ses services ou Bernie Rose qui est le véritable propriétaire du garage et un parrain de la mafia locale). Puis, progressivement, elle sort du personnage, comme en attestent les scènes capitales que sont celle du diner, celle du braquage raté, celle de l'interrogatoire du sbire de Nino et, bien sûr, celle de l'ascenseur. Ces scènes permettent au réalisateur de présenter en crescendo la violence inhérente (mais jusque là insoupçonnée) de Driver. D’une dimension simplement verbale, il passe à l'utilisation de la violence physique et armée qui va crescendo. D'abord, pour se défendre, il utilise une tringle de rideau de douche, puis, pour obtenir des informations, il a recours à un marteau, avant d'atteindre un paroxysme de folie meurtrière : il tue à coups de pied, puis s'acharne sur le cadavre de l'homme que Nino a envoyé pour se débarrasser d'eux.

Si la découverte de cette facette se fait de manière progressive et cumulative pour le spectateur, il n'en va pas de même pour Irene pour qui la révélation est brutale. Il n'est sans doute pas inutile de souligner que l'impact de cette révélation est accentué par le fait qu'elle se produit immédiatement après le premier contact physique (une étreinte et un baiser) entre les deux personnages. La coïncidence de ces pôles opposés semble déterminer Driver comme héros tragique, qui ne peut échapper à la malédiction qui l'afflige et le condamne à rester solitaire. Dans cette perspective, le rappel à son statut est d'autant plus violent qu'une intimité extrêmement "pure" est démontrée.

La prise de conscience de la monstruosité cachée de celui qu'elle pensait être un digne successeur à son mari - mais aussi un héros protecteur - est un moment de cinéma remarquable par son économie: incrédule et pleine d'effroi, Irene recule pour s'extraire de l'ascenseur et de l'horreur de ce qui se déroule sous ses yeux. La séquence s'achève, en trois plans, sur la circulation des regards qui déterminent la dernière image/vision/identité que vont avoir les deux protagonistes l'un de l'autre. Le premier plan épouse le point de vue d'Irene : Driver est de dos, puis il se retourne lentement et la regarde d'un air à la fois perdu et encore plein de la rage dont il vient de faire la démonstration. L'éclairage de la scène, la lenteur du mouvement et la posture légèrement voutée de Ryan Gosling suggèrent une difformité et une malfaisance qui contrastent radicalement avec le physique et les manières angéliques que nous lui avons vus jusqu'alors, comme s'il venait de révéler le côté Hyde de sa personnalité ordinairement Jeckyll. Le plan suivant est le contre-champ du précédent: Irene, hébétée, immobile, fixe l'indescriptible hors champ jusqu'à ce que les portes de l'ascenseur se referment. Le dernier plan de la séquence est un gros plan sur le scorpion brodé ornant le dos du blouson que porte Driver. ${ }^{9}$ Le transfert qui s'opère dans cette scène entre la parole et l'image (absence de la première au profit de la seconde) intime l'incommunicabilité qui résulte de la crise de violence, tant pour les personnages entre eux que pour le spectateur : quelque chose d'irréparable s'est produit et la fragilité de 
la relation entre Irene et Driver ne saurait survivre à l'overkill dont nous venons d'être témoins. En parallèle et de manière complémentaire, le paradoxe de l'acte (violent et pourtant salvateur) engage le spectateur à saisir la portée symbolique (et elle aussi paradoxale) de l'image du scorpion, et sur ce qu'elle figure de la nature de Driver. Notre perception du personnage en est profondément modifiée. Il a franchi une limite qui le positionne au delà de ce qui est moralement justifiable. L'importance de ce moment de basculement entraîne également une réévaluation du statut de héros de Driver.

Ainsi, comme chez Kubrick à nouveau, la violence condamne le protagoniste à rester à l'extérieur de ce qui lui semble être désirable (l'amour d'une femme, l'intégration à une structure familiale...) tout autant qu'elle le contraint à aller jusqu'au terme de son engagement, jusqu'à la résolution du paradoxe de son identité. Homme multiple (comme le signalent les effets de reflet de son image présents tout au long du film), il est aussi homme sans visage, tant littéralement que métaphoriquement. L'aspect lisse $\mathrm{du}$ visage de l'acteur, et le masque en latex (aux traits informes, sans chevelure et qui lui couvre la tête et le haut des épaules) dont il se sert pour ses activités de cascadeur (autrement dit de doublure invisible d'un autre acteur) annulent en quelque sorte la distinction d'une identité. C'est donc par ses actions que Driver peut accéder à une autre fonction, à une autre identité. Comme le laisse entendre l'un des sens du nom commun anglais "drive ", le protagoniste est "irrépressiblement poussé » à circuler (faisant alors corps avec son véhicule), mais aussi à se transformer, à muter, en passant du statut d'homme-machine à celui de héros. ${ }^{10}$ Cette relation dialectique entre violence et identité n'est pas une innovation pour le réalisateur. Bien au contraire, elle parcourt thématiquement et esthétiquement l'œuvre de Refn pour qui, de la trilogie Pusher (1996, 2004, 2005) jusqu'à Valhalla Rising (2009) en passant par Bronson (2008), tout est une question de transformation. ${ }^{11}$ Après le visionnage des films en question, il apparaît comme une évidence au spectateur que la "transformation" dont fait état Refn combine une (des) modification(s) physique(s) et/ou symbolique(s) pour le personnage ainsi qu'un changement dans le regard qui est porté sur le personnage, à la fois par les protagonistes intradiégétiques et par le spectateur lui-même. La métamorphose engagée, le personnage peut quitter ses attributs de simple individu pour endosser, symboliquement, les atours du héros. Et si une référence à l'univers des justiciers masqués et costumés devait être évoquée, il faudrait sans doute se tourner vers la figure de Batman qui sait qu'il est un mal nécessaire pour assurer le bon fonctionnement de Gotham City. Cet aspect-là des créations du cinéaste correspond à sa volonté de ne présenter que des "moments» de la vie de ses personnages : pris in medias res, leur parcours ne peut que rester en suspens, puisque c'est à destination du spectateur que le processus de transition est mis en scène ; c'est lui qui doit remplir l'avant et l'après.

Si circulation d'identité il y a, il n'est pas inenvisageable de concevoir les deux films en terme d'ouverture et d'appropriation qui manifestent ainsi un autre type de circulation, conçu ici comme forme d'échange.

28 Le genre très codé du film de course déjà à l'œuvre dans Cars $^{12}$ est supplanté et complété, avec Cars 2, par les codes (intrigues, motifs, personnages, musique...) de l'espionnage ${ }^{13}$ en images. John Lasseter et Brad Bird revendiquent l'influence de la série The Man From U.N.C.L.E. ${ }^{14}$ mais également de films plus récents, comme la trilogie Jason Bourne, au point de rendre hommage à leurs modèles en demandant à Michael Giacchino de trouver un thème musical «à la Bond » ou à Michael Caine de donner voix 
à l'agent secret Flinn McMissile. ${ }^{15}$ De sorte qu'aux changements de décors dictés par l'intrigue viennent se greffer des personnages qui fleurent bon les adaptations des romans de Ian Fleming (tels ces méchants affublés de monocle ou obsédés par leur volonté de domination du monde), voire ceux de Mario Puzo (à cet égard, la réunion des chefs des différentes mafias de tacots dans un casino est digne du Godfather). Un des apports majeurs du travail des deux réalisateurs est sans conteste Holley Shiftwell. Certes, le personnage possède une dimension stéréotypée (de part sa couleur, son inexpérience du terrain, son émancipation tardive dans le film...) et l'on peut sans doute faire grief aux créateurs de la franchise d'être en décalage par rapport aux réalités socio-économiques contemporaines où les femmes sont pilotes et mécaniciens, et de perpétuer la dimension phallocentrique du monde cinématographique de l'automobile. Il n'en demeure pas moins que cette figure féminine au caractère trempé et prodige en matière de technologies modernes, qui seconde magistralement l'espion aux multiples talents et aux gadgets non moins nombreux, apporte une touche de féminité bienvenue dans cet univers fortement dominé par l'élément masculin. D'autant qu'il convient d'indiquer qu'elle concentre bien plus de caractéristiques positives que l'ensemble des «figures » féminines présentes dans Cars.

Quant à lui, Drive est à la croisée de plusieurs influences. Film de commande (à l'instigation de la star Ryan Gosling $\left.{ }^{16}\right)$, il s'intègre, d'une part, de manière évidente au film noir américain, mais aussi au film criminel européen (lui-même influencé, bien sûr, par le film noir) dont Jean-Pierre Melville (et son film emblématique Le Samouraï de 1967) est la figure tutélaire. D'autre part, il est modelé par la personnalité et les goûts propres du metteur en scène danois. En effet, parmi ses sources d'inspiration, ce dernier (qui, pour l'anecdote, n'est pas détenteur d'un permis de conduire...) évoque l'approche fétichiste des véhicules à moteur ${ }^{17}$ qui conditionne l'esthétique des productions de Kenneth Anger (Scorpio Rising, 1969, et Lucifer Rising, 1972) autant que l'utilisation des miroirs (The Lady from Shanghai, 1947) ou des personnages noirs et complémentaires singulièrement jusqu'au-boutistes comme ceux de Touch of Evil (1958) dans le cinéma d'Orson Welles, ou encore l'Irréversible (2002) de Gaspard Noé pour la scène de meurtre (avec un extincteur, dans le film modèle).

Ce que révèlent ces références, à tout le moins, c'est que les travaux des différents cinéastes proposent, chacun à sa manière, des formes de circulation culturelle. Pour sa part, la réalisation de Lasseter et Bird délocalise ce qui était dans le film précédent étroitement américain (le monde de la compétition NASCAR, mais aussi l'univers de la petite ville revisitée à l'aune de la pastorale américaine) en lui faisant parcourir le globe. Le générique de fin de Cars 2 illustre parfaitement cette apparente volonté de croisements et d'échanges culturels liés à l'ouverture sur le monde puisque, accompagnés par la chanson (au titre évocateur) Collision of Worlds, que le britannique Robbie Williams interprète en duo avec le chanteur de country américain Brad Paisley, l'on voit les protagonistes, en images animées à l'esthétique plus grossière que celles du reste du film, circuler autour de la mappemonde et visiter les lieux mythiques de quelques douze pays : de Buckingham Palace à l'opéra de Sydney, du Taj Mahal à la tour Eiffel, les monuments sont rendus sur des imitations de cartes postales. Mais qu'on ne s'y trompe pas : tout en prenant en compte le public (essentiellement enfantin) auquel le film est destiné, il est intéressant de noter que Cars 2 poursuit, à une échelle plus large, l'entreprise entamée en 2006 avec Cars. Dans le même temps où le film évacue complètement la figure humaine (ainsi que nous l'avons déjà souligné), il promeut et renforce une vision américaine du monde. De sorte que l'on peut aisément défendre 
l'idée selon laquelle ce à quoi le spectateur est exposé ressemble moins à une véritable circulation culturelle qu'à une circulation unilatérale qui révèle le point de vue (imaginaire, fantasmé?) des États-Unis sur le reste du monde.

31 De fait, à l'instar de la majeure partie des productions d'outre-Atlantique destinées à toucher un public aussi large que possible, les personnages et les lieux archétypaux évoqués plus haut subissent les déformations résultant d'une lecture au prisme d'Hollywood: clichés, inexactitudes et invraisemblances se cachent derrière la licence poétique, comme pour mieux réaffirmer un modèle américain reposant sur des valeurs consensuelles, en tête desquelles on retrouve l'indéfectibilité de l'amitié et la nécessité vitale de se reposer sur un modèle familial (quand bien même il s'agirait d'une famille de substitution). On peut également s'interroger légitimement sur l'impossibilité pour Mater de nouer une relation durable avec Holley. Justifiée, au moins en partie, par leurs activités professionnelles respectives, leur histoire d'amour impossible relève tout autant de cette vieille tradition hollywoodienne qui tend à condamner les mélanges (de race, comme de culture). En ce sens, les implications idéologiques de la séparation (chacun chez soi et entre gens du même monde) correspondent à ce qui a été déjà observé concernant les formes d'autarcie, de circuit fermé, de boucle dans le film.

Néanmoins, deux caractéristiques de Cars 2 se révèlent plus troublantes à la lecture attentive du texte filmique. Il s'agit, d'une part, de la résolution de l'intrigue qui rend naturelle (et rapide) la collaboration entre la société civile et les forces armées : c'est en partie grâce à l'intervention personnelle d'un personnage secondaire (la jeep / le Sergent fait appel à un ami de son père qui fait partie de l'armée britannique) qu'il est mis un terme aux activités des tacots. Dans le contexte réel de relations internationales dont la complexité le dispute à la défiance vis-à-vis des interventions militaires conjointes, il n'est pas impossible d'imputer le rôle positif que le film attribue à l'influence dont semble jouir l'armée US auprès de Londres à l'image que se font les US de l'Angleterre.

Deuxième élément remarquable, c'est ce même Sergent (dont l'autorité et le mépris pour les marginaux a été établie dans Cars) qui introduit une part de subversion (peutêtre sous l'influence du mini-van Volkswagen hippy Fillmore) dans un discours plutôt conservateur sur les énergies vertes (le carburant biologique développé par Axelrod n'étant finalement qu'une escroquerie). Contre toute attente, c'est bien lui (et non Fillmore, comme on était en droit de s'y attendre) qui a fourni (à son insu) à McQueen la production locale de Radiator Springs et lui a ainsi permis de surmonter toutes les épreuves. En guise de justification à ce qui semble être une contradiction avec les valeurs qu'il est sensé défendre, il formule l'aphorisme suivant : «Once big oil, always big oil...man !» (« Quand on fait partie des gros producteurs de pétrole, c'est pour la vie... mon pote $! ») .^{18}$ Même chez les plus conservateurs des personnages, il reste un fond de défiance vis-à-vis d'une forme d'affairisme dévoyé, heureusement incarné (l'autocritique est tenue à distance) par un aristocrate anglais. Ce qui correspond, on en est bien conscient, à la confortable tendance actuelle de ne pas critiquer le capitalisme, mais ceux qui l'utilisent à mauvais escient.

Le spectateur, même enfantin, perçoit ce qu'il y a de rassurant et protecteur (de protectionniste, pourrait ajouter l'observateur critique) dans cette illustration d'une morale préservée («tout est bien qui finit bien») prolongée par le retour au bercail final. Un message aux accents émersoniens peut s'entendre dans la volonté de Mater de se contenter de son chez soi, malgré la sollicitation des deux espions britanniques. La 
perspective de reprendre une vie paisible l'emporte sur les louanges et la promesse d'autres aventures aussi trépidantes qu'internationales.

De son côté, Drive est le premier film américain d'un réalisateur européen qui n'est pas étranger à la culture américaine, puisqu'il a passé près d'une dizaine d'années à New York où il a étudié à l'American Academy of Dramatic Arts. Dans la lignée de cinéastes européens venus exercer leur talent à Hollywood sous l'impulsion d'une star américaine, Refn peut comparer sa collaboration avec Ryan Gosling à celle qui a amené Lee Marvin à être dirigé par John Boorman dans Point Blank (1967) ou à la demande expresse de Steve McQueen d'obtenir la participation de Peter Yates sur Bullitt (1968). ${ }^{19}$ De plus, sa filmographie comme les entretiens qu'il a accordés laissent peu de doute quant à sa connaissance du cinéma américain.

Dès lors, le travail de Nicolas Winding Refn ne saurait être limité à une ode à l'un des piliers de la culture populaire américaine. Certes, le projet d'origine, qu'il décrit comme « un film d'action de 60 millions de dollars, un homme en fuite au volant d'une voiture [...] écrit pour Hugh Jackman, avec des types musclés et des cascades $»^{20}$, lui aurait permis de s'aligner sur d'autres productions comme la série des Fast and Furious. ${ }^{21}$ Mais il est plus significatif de retrouver dans le résultat final des éléments caractéristiques de deux fleurons éminemment américains du cinéma de genre : le film noir et le western. Et, à la confluence de ces deux configurations narratives et esthétiques, le personnage de l'homme solitaire et taciturne qui, par choix ou par nécessité, s'impose comme la figure fondamentale du vecteur de la violence, et dont Driver incarne une variation. Pour sexiste, misogyne et phallocrate que soit ce modèle de héros masculin, le cinéaste n'en conserve que la caractéristique commune, c'est-à-dire son rapport à la violence, afin de continuer à creuser une réflexion sur cette dernière entamée dès ses premières réalisations. Cependant, s'appuyant sur une longue tradition de vengeurs solitaires (dont les figures incarnées par Clint Eastwood et Charles Bronson sont les archétypes), Refn insuffle une dimension métaphysique ignorée par ses modèles. De manière paradoxale mais révélatrice, le mutisme (relatif) du protagoniste lui confère une profondeur qui fait défaut à « Dirty » Harry Callahan ou à Paul Kersey : il ne dit rien sur ce qui le motive et sur ce qui le pousse à avoir recours à une violence disproportionnée. Cette forme d'existentialisme minimaliste ne correspond guère à une vision américaine du héros vengeur qui se doit de justifier ses actions.

C'est en insistant sur le paradoxe inhérent au personnage que Refn nous amène à pénétrer dans son univers. D'une part, il y a la description d'une parenthèse enchantée (la scène où Driver emmène Irene et son fils en balade pour trouver un îlot de nature au cœur même de la ville) qui correspondrait (jusque dans l'esthétique clichée de la séquence) à la réalisation d'un rêve américain archétypal (Eden inclus). Mais l'artifice fait long feu puisque le paradis n'est qu'une impasse (littéralement et métaphoriquement), temporairement atteint et qui finalement contraint le personnage à faire demi-tour. La vie au sein d'une famille nucléaire (même recomposée) n'est pas son destin - mais quel héros solitaire (et archétypal) peut envisager un tel renversement de situation?

D'autre part, il y a, ainsi que nous l'avons vu, cette violence innée chez Driver qui en fait un personnage aliéné (au sens médical comme au sens social) et autodestructeur qui ne doit son salut, sa rédemption qu'à l'explosion de violence qui protège la veuve et l'orphelin et purifie (temporairement, au moins) la ville de Los Angeles tout en l'excluant de manière irrémédiable du tissu social (comme tous les autres personnages 
développés ailleurs par Refn). Si cette dimension du protagoniste n'est pas en soi novatrice, elle permet au moins à Refn d'aborder pour la première fois l'imaginaire américain par le biais d'une figure iconique, comme l'étaient, dans des contextes culturels différents, Bronson ou One-Eye ${ }^{22}$ tout en préservant, ainsi qu'il a été montré, ce qui différencie fondamentalement ces personnages de leurs partenaires nord américains : leur rapport au langage.

«A Real, Real Hero » répète le groupe College. Ou encore un super-héros, si l'on en croit l'acteur et le metteur en scène. ${ }^{23} \mathrm{Et}$, de fait, Driver correspond à ce personnage coincé entre deux mondes, entre deux états, entre l'ombre et la lumière. Un personnage dont le véhicule lui permet de réaliser des prodiges, dont le vêtement réversible lui sert tout à la fois de déguisement et d'armure. Bref, un personnage mythique qui n'est pas sans évoquer le super-héros venu du monde de la bande dessinée et qui peut sans doute s'articuler avec la théorie du monomythe américain développée par John Shelton Lawrence et Robert Jewett. ${ }^{24}$ Selon eux, l'histoire archétypale de tout héroïsme se déroule selon le schéma suivant : une communauté en parfaite harmonie est menacée par un danger; les institutions normales sont impuissantes à y faire face; un héros désintéressé apparaît, renonce à toutes les tentations et assume la tâche rédemptrice ; aidé par le sort, sa victoire décisive restaure la communauté dans sa perfection originelle: le héros retourne à l'obscurité. Drive illustre cette définition et Driver son personnage central, tout en apportant une modulation sous la forme de l'intérêt amoureux qui semble motiver son intervention. ${ }^{25}$

Tous les éléments qui ont été ici évoqués permettent d'apprécier l'articulation des deux films analysés dans la problématique de la circulation. Si le sous-genre que représente le film d'automobile est un vecteur idéal pour aborder cette notion, Cars 2 et Drive proposent des inflexions qui tiennent à la fois de la continuité, mais aussi de la singularité. D'une certaine manière ni l'un ni l'autre ne sont révolutionnaires : le premier est compris avant tout comme une suite, le second ne met pas en scène de personnage véritablement inédit. De plus, ils s'intègrent tous deux dans une continuité d'un autre ordre : Cars 2 appartient de plein droit à la série des films d'animation prolongeant le travail de la firme Disney, d'une part, et, d'autre part, Drive permet à son réalisateur de poursuivre son travail sur la relation entre masculinité et violence.

41 Cependant, l'originalité du film de Nicolas Winding Refn réside dans la stylisation de son traitement du sujet. Sa réappropriation des codes d'un genre éprouvé lui permettent de transformer sa matière première en une expérience singulière. La circulation du sens est aussi pour le cinéaste une circulation des sens lorsqu'il transfère, de manière presque synesthésique, le ressenti des personnages vers d'autres vecteurs que la parole. Ainsi l'exacerbation des détails sensoriels portés par la musique, l'éclairage ou la chromographie permettent de compenser le laconisme de Driver.

Quant à l'originalité du fonctionnement du monde de Cars, sur le modèle du "tout véhicule", rappelons-le, elle a continué à fonctionner pour une série de court-métrages qui mettent scène Mater ${ }^{26}$ Malheureusement, la transposition du procédé à un autre support (des voitures aux avions pour Planes, 2013) n'a pas été reçue avec le même enthousiasme que son prédécesseur. ${ }^{27}$ À trop vouloir dupliquer une recette sans y ajouter une dose d'originalité significative, l'écurie Pixar paraît avoir épuisé jusqu'à son public cœur de cible. Une circulation qui semble, au moins temporairement, à l'arrêt. 


\section{BIBLIOGRAPHIE}

Cars 2, John Lasseter - Brad Lewis, Walt Disney Pictures, Pixar Animation Studios, 2011.

Ciment, Michel et Yann Tobin. « Entretien avec Nicolas Winding Refn » in Positif $n^{\circ} 608$, Octobre 2011, 9-15.

Drive, Nicolas Winding Refn, Bold Films, Odd Lot Entertainment, Marc Platt Productions, Seed Productions, 2011.Gilchrist, Todd. « Under the Hood » in Boxoffice, Vol.147, Issue 6, June 2011.

http://artsbeat.blogs.nytimes.com/2011/05/22/cannes-q-and-a-driving-in-a-noir-l-a/

http://www.lesinrocks.com/2011/10/05/cinema/nicolas-winding-refn-rencontre-avec-unenfant-terrible-du-cinema-de-genre-118668/

Koelher, Robert. « And the Search for a Real Hero » in Cinema-Scope Magazine, October 2011, 6-10.

Lawrence, John Shelton and Robert Jewett. The Myth of the American Superhero. Grand Rapids :

William B. Eerdmans Publishing Co, 2002.

McDonagh, Maitland. « Drive » in Film Journal International, Vol. 114, Issue 10, Oct 2011.

McLean, Thomas J. « Mr. Lasseter's Wild Ride » in Animation Magazine, June 2011, 4-6.

Roddick, Nick. « Stealer's Wheels » in Sight \& Sound, Vol. 21, Issue 10, Oct 2011, 46-48.

Rouyer, Philippe. « A Real, Real Hero » in Positif n608, Octobre 2011, 7-8.

Schwarzbaum, Lisa. « Drive » in Entertainment Weekly, Issue 1173, 9/23/2011.

Solomon, Charles. « All Revved Up ! » in Animation Magazine, July 2006, 10-12.

Witmer, Jon D. « Road Warriors » in American Cinematographer, October 2011, 28-43.

Cars

\section{NOTES}

1. Pour en avoir un aperçu: myloveofoldhollywood.blogspot.fr/2011/01/stars-and-theirfabulous-cars-part-4.html

2. Herbie Rides Again (Un Amour de coccinelle), Robert Stevenson, 1974 ; Herbie Goes to Monte Carlo (La coccinelle à Monte Carlo), Vincent McEveety, 1977 ; Herbie Goes Bananas (La coccinelle au Mexique), Vincent McEveety, 1980 et Herbie Fully Loaded (La coccinelle revient), Angela Robinson, 2005.

3. On trouvera un grand nombre d'exemples de ces distorsions sur la page du film: http:// www.imdb.com/title/tt1216475/trivia?ref_=tt_trv_trv

4. Successivement, dans le déroulement de la narration, il aide des cambrioleurs à échapper à la police, il répare des voitures dans un garage, il réalise des cascades automobiles pour le cinéma, il prépare une course de GT, il promène sa voisine et son petit garçon, il fuit, il tue, il disparaît.

5. Voir l'entretien croisé du metteur en scène et de son acteur principal, Mads Mikkelsen, en bonus dans l'édition DVD de Valhalla Rising, 2009.

6. Ma traduction.

7. Idem.

8. «There are thousands of streets in Los Angeles » - « Il y a des milliers de rues à Los Angeles. » Ibidem. 
9. Toute la charge symbolique de l'animal est alors rendue cruellement évidente : l'ambivalence du personnage est signalée par la réversibilité du blouson (un côté blanc, l'autre sombre) et le scorpion phallique associe amour et mort (pôles opposés déjà présents dans la relation mythologique d'Orion et de Diane) tout autant qu'il incarne l'irrépressible instinct de tuer, comme le rappelle la fable du scorpion et de la grenouille, évoquée ailleurs dans le film.

10. La chanson A Real Hero (College\&Electric Youth) qu'on entend ponctuellement tout le long du film, ne dit rien d'autre.

11. Koeler 9 .

12. Et dont on pouvait craindre que Cars 2 ne soit qu'une simple redite.

13. Avec l'univers de l'automobile, c'est l'autre passion avouée de Lasseter depuis l'enfance.

14. Cette série de 105 épisodes a été diffusée sur la chaîne de télévision américaine NBC entre le 22 septembre 1964 et le 15 janvier 1968.

15. L'acteur a incarné Harry Palmer (l'espion créé par Len Deighton en 1962) à cinq reprises.

16. Le réalisateur raconte avec force les détails de la genèse du projet dans les bonus de l'édition DVD du film.

17. Ce qui peut sembler paradoxal et provocateur pour quelqu'un qui n'a pas le permis de conduire et ne possède pas de voiture.

18. Ma traduction.

19. Ciment et Tobin 11.

20. Id 9.

21. Cette série de blockbusters, vehicles pour la star Vin Diesel, a débuté en 2001 et comprend, à ce jour, six éléments, un septième étant déjà annoncé pour avril 2015 (http://www.imdb.com/ title/tt2820852/?ref_=nv_sr_1).

22. Respectivement, les protagonistes du film éponyme de 2008 et de Valhalla Rising en 2009.

23. http://artsbeat.blogs.nytimes.com/2011/05/22/cannes-q-and-a-driving-in-a-noir-l-a/, et http://www.lesinrocks.com/2011/10/05/cinema/nicolas-winding-refn-rencontre-avec-unenfant-terrible-du-cinema-de-genre-118668/

24. Lawrence, John Shelton and Robert Jewett. The Myth of the American Superhero. Grand Rapids : William B. Eerdmans Publishing Co, 2002.

25. Ce film serait-il une compensation pour le film de super-héros que le réalisateur danois aurait rêvé de tourner? Il y a eu, en effet, beaucoup de spéculations autour d'un projet d'adaptation des aventures de l'une des icones de la firme DC Comics, avec Christina Hendricks dans le rôle de Wonder Woman et Nicolas Winding Refn derrière la caméra.

26. Mater and the Ghostlight, 2006 ; Tokyo Mater, 2008 ; Cars Toons : Mater's Tall Tales, 2010, par exemple.

27. Lors du premier week-end d'exploitation, Cars 2 a rapporté 66135507 dollars, contre 22232 291 dollars à son successeur. Source : http://boxofficemojo.com, consultée le 15 octobre 2013.

\section{RÉSUMÉS}

Bien qu'ils soient apparemment très différents, Cars 2 et Drive s'intègrent dans une phénoménologie américaine de l'automobile et la problématique de la circulation - au sens littéral comme au sens figuré - permet d'observer ce que les deux films ont à dire sur un certain état de l'Amérique et de son idéologie en ce début du vingt-et-unième siècle. 
Though they may appear as widely different, Cars 2 and Drive belong to the American cultural cornerstone of cars. The theme of traffic - to be taken both literally and figuratively - appears as an apt and revealing means to observe a certain ideological status of the United States in the early twenty first century.

INDEX

Keywords : automobiles, traffic, hero, United States, ideology

Mots-clés : automobiles, circulation, héros, Etats-Unis, idéologie

\section{AUTEURS}

\section{YANN ROBLOU}

Université de Valenciennes

Maitre de conférences

yann.roblou@univ-valenciennes.fr 